\title{
A transcranial Doppler sonography study of shoot/don't-shoot responding
}

\author{
Natasha B. Schultz \\ Georgia State University, Atlanta, Georgia \\ GERALD MatThews \\ University of Cincinnati, Cincinnati, Ohio \\ JOEL S. WARM \\ Air Force Research Laboratory, Wright-Patterson Air Force Base, Ohio \\ AND \\ DAVID A. WASHBURN \\ Georgia State University, Atlanta, Georgia
}

\begin{abstract}
The purpose of this study was to examine the relationship between changes in cerebral blood-flow velocity and performance on a speeded shoot/don't-shoot task. Brain activity as indicated by cerebral blood-flow velocity (hemovelocity) was recorded using the transcranial Doppler ultrasonography. A shoot/don't-shoot decisionmaking task presented participants with threat/nonthreat stimuli in the form of bull's-eye images of various colors. Participants were required to shoot threat targets using a laser-modified handgun. Results support a vigilance decrement in both the performance measures and hemovelocity. Performance, as measured by reaction time, number of hits, and marksmanship, decreased across the length of the vigil. Hemovelocity slowed across the left and right hemispheres as the task progressed, and hemovelocity was slower in the right hemisphere than in the left hemisphere.
\end{abstract}

Vigilance, or sustained attention, requires a person to maintain attention on a region of interest for a prolonged time period (See, Howe, Warm, \& Dember, 1995). A broad range of real-life tasks require the ability to sustain attention to detect an object or signal of interest. For example, airport security screeners monitor X-ray images of suitcase after suitcase to detect the occasional prohibited item. Soldiers guarding an area need to be attentive to possible threats in the region. Students listening to a bad lecture need nonetheless to detect the facts that might appear on an exam. These examples of vigilance are only a few of the many tasks that rely heavily on this ability to remain vigilant under circumstances that may be boring and where target events may be infrequent.

Over the years, vigilance has been extensively studied. An adequate review of this literature is beyond the scope of the present article (but see Parasuraman, 2000; Parasuraman \& Davies, 1984; See et al., 1995). Generally, however, attention does not stay at the optimum level across timeon-task: Performance on vigilance tasks declines across the vigil (Mackworth, 1961). This is known as the vigilance decrement. Half of the reduction in signal detection performance occurs within the first $15 \mathrm{~min}$ of a vigilance task, and the decrement has reached the lowest performance within
20-35 min (Teichner, 1974). Current research suggests that performance decreases as quickly as 5-10 min after task onset (Helton, Dember, Warm, \& Matthews, 1999). In a sense, the difficulty of boring, low-target-frequency vigilance tasks is the mental effort that must be expended to maintain attention when nothing about the task elicits attention exogenously (Hart \& Staveland, 1988).

Having defined what performance looks like when a participant is inattentive, the present research focus has turned to two questions: "Are there behavioral or physiological indicators that a participant is about to become inattentive?" and "If so, what can be done about it?" These were the questions that motivated the present applicationinspired research and our efforts to see whether a particular psychophysiological marker would be useful for diagnosing and ameliorating inattention-before it has an effect on performance.

Psychophysiological research utilizes different physiological measures to complement behavioral data found in the research. In the present study, we focused on a physiological measure that records brain activation as an indication of task-induced mental workload in each of the cerebral hemispheres. Researchers have used functional transcranial Doppler (TCD) sonography to investigate

D. A. Washburn, dwashburn@gsu.edu 
changes in cerebral blood-flow velocity (BFV) related to performance of a number of visual tasks, including vigilance (Hitchcock et al., 2003; Warm \& Parasuraman, 2007); however, TCD has not been used to try to predict sustained performance in a shoot/don't-shoot (S/DS) judgment task.

\section{TCD Sonography}

TCD is a noninvasive procedure that uses ultrasound technology to continuously measure cerebral BFV through main stem intracranial arteries in the brain (Aaslid, Markwalder, \& Nornes, 1982). The three major arteries that can be measured are the middle cerebral artery (MCA), posterior cerebral artery (PCA), and anterior cerebral artery (ACA).

Transducers (ultrasound probes) send a Doppler ultrasound signal through the transtemporal window of the skull to the specified artery and receive that signal; thus, the transducers work as both the source and receiver. Doppler sonography depicts a frequency shift caused by the movement between the source and receiver (Duschek \& Schandry, 2003). In the arteries, erythrocytes (red blood cells) in the blood cause the frequency shifts picked up by the ultrasound signal. The cerebral BFV is proportional to the size of the frequency shift. The transtemporal window is one of three areas in the skull that are thin enough to allow the ultrasound signal to penetrate the bone. Each artery displays different average velocities and directional flow, which allow for verification of the artery. The artery that was the focus of this study was the MCA, since it profuses to approximately $80 \%$ of the brain to supply blood to the anterior, middle, and posterior temporal arteries, the anterior and posterior parietal arteries, and the lateral orbitofrontal and ascending frontal arteries (Netter, 1989).

The MCA profuses most of the lateral surface of the brain (Angevine \& Cotman, 1981), thus covering the lateral part of the frontal, parietal, temporal, and occipital cortices of the brain (Hanaway, Woolsey, Gado, \& Roberts, 1998). Examples of the specific parts of the brain fed by the MCA are the lateral portions of orbital gyri, large parts of precentral and postcentral gyri, superior and inferior parietal lobules, superior and middle temporal gyri temporal pole, and motor and premotor areas. The MCA also extends caudally to supply most of the lateral gyri of the occipital lobe. The intraparietal sulcus divided the portions of parietal lobe caudal to postcentral gyrus into superior and inferior parietal lobules (Carpenter, 1976).

Large basal arteries, such as the MCA, change their cerebral BFVs as an effect of the demand in the brain regions that are serviced (Duschek \& Schandry, 2003). Changes in cerebral activation and metabolism initiate changes in the cerebral BFV. As the cerebral activation increases, the brain area engaged requires larger amounts of oxygen, removal of waste, and local increase in $\mathrm{pCO}_{2}$ (Stroobant \& Vingerhoets, 2000). Therefore, as the workload increases in brain regions, BFV to those regions increases. For example, visual and verbal tasks significantly increase BFV from baseline conditions (Bulla-Hellwig, Vollmer, Götzen, Skreczek, \& Hartje, 1996; Cupini et al., 1996; Silvestrini, Cupini, Matteis, Troisi, \& Caltagirone, 1994).
Early research that has used TCD to measure BFV has focused on hemispheric differences in various verbal and visual tasks (Bulla-Hellwig et al., 1996; Cupini et al., 1996; Silvestrini et al., 1994). However, more recently, TCD has been used to investigate hemispheric differences in a broadening array of cognitive tasks. Vigilance research has previously incorporated TCD to look at changes in activation across the task. BFV declined across a vigilance task that required observers to watch for a small reduction in the size of a recurring grating (Schnittger, Johannes, Arnavaz, \& Munte, 1997). Previously the vigilance decrement was affiliated with declining BFV (Hitchcock et al., 2003; Warm \& Parasuraman, 2007). Reliable cuing removed the drop of BFV across the vigil (Hitchcock et al., 2003). Additionally, BFV was lateralized in such a way that the right hemisphere was greater than the left hemisphere (Hitchcock et al., 2003; Schnittger et al., 1997; Warm \& Parasuraman, 2007).

There are benefits of this technology that make it appropriate for the present study. TCD enables the manipulation of the S/DS task to see the relationship between performance and brain activation as reflected in changes in cerebral BFV. BFV shows brain activity as a function of the elements of the task (Duschek \& Schandry, 2003). The larger the workload, the higher the BFV will be in the brain. A secondary concern for choosing TCD in this study was the advantage of TCD in the practical uses. It is more cost effective than FMRI, and application of the TCD and use during testing causes much less stress on participants than fMRI. More important, this technology enables the participants to move freely and shoot a gun at multiple targets, because a helmet holds the transducers in position to enable movement. TCD allows comparison between the levels of brain activation in each hemisphere. Finally, TCD provides continuous measures of BFV throughout the task. TCD has relatively good temporal resolution, showing BFV changes from visual stimulation as short as $34 \mathrm{msec}$ (Duschek \& Schandry, 2003). The temporal resolution enables the psychophysiological research paradigms to resemble those used in the research that looks at only behavioral data.

Motivated by an applied interest from our funding agent in predicting inattentive states, TCD enabled us to conduct use-inspired basic research on the relation between brain activation and performance in a vigilance task. That is, we were not interested in attentiveness or even brain activity per se, but rather were interested in TCD itself as a diagnostic instrument. We predicted that BFV would be able to predict error and slow responding in S/DS performance. In addition to this prediction, we explored hemispheric differences across the task and in each type of response. Furthermore, we predicted that changes in brain activation across hemispheres would indicate changes in performance.

\section{METHOD}

\section{Participants}

Participants (120 female, 27 male; 4 preferred not to answer) from the Georgia State University research participant pool participated in this study. Ages ranged from 18 to 44 years, with a mean of approximately 19 years. All participants were right-handed and had normal or corrected-to-normal (contacts) vision. Participants who 
wore glasses were excluded, because the frame of eyeglasses interfered with the ability to acquire a signal with the apparatus. For the present analyses, we included only those participants for whom a reliable and stable signal for TCD (see below) could not be obtained for both hemispheres. A consistent signal was determined by the waveform of the signal. If the waveform was the same from heartbeat to heartbeat, then it was stable and consistent. The participant had to be able to turn her or his head from side to side slowly without the signal disappearing or becoming unstable. If it was determined that a signal could not be obtained in both hemispheres, the participant was debriefed and allowed to leave. All participants received 2 research-credit hours for their participation, regardless of whether the signal was obtained. After these exclusion criteria, 38 volunteers were included in the present analyses.

\section{Apparatus and Procedure}

Upon arrival, the participant completed a consent form and optional demographics form. The Edinburgh handedness inventory (Oldfield, 1971) was then completed to verify the right-handedness of the participant. The experimenter then verbally explained the apparatus being used and the directions for the task. After verifying that the participant had no questions, the experimenter proceeded with setting up the equipment to find hemovelocity. We measured hemovelocity by averaging cerebral BFV across a 2-sec interval. Participants were tested individually, with the experimenter present during all phases to verify the continual functioning of all equipment and continual comfort of the participants. Each participant calibrated the laser-modified handgun. After completing the Peacekeeper task (described below), participants were debriefed and thanked for their participation. The experimenter verified that the participants had no further questions, and they were dismissed.

TCD sonography. The Companion III TCD unit (Nicolet/EME, Madison, WI) measured hemovelocity. WinTCD software recorded the mean hemovelocity in both hemispheres. The participant was fitted with a Welder TCD headband that held two 2-MHz ultrasound transducers steady at the transtemporal window located at the temples, as seen in Figure 1A. A small amount of Aquasonic-100 ultrasound transmission gel was applied to the face of each transducer and to the skin at the transtemporal windows to enhance the transducer's signal reception.

Hemovelocity was measured in the MCA in both hemispheres and was recorded at depths of $48-56 \mathrm{~mm}$, which was measured as the distance between transducer face and sample volume. The recording depth was adjusted by $2-\mathrm{mm}$ increments to compensate for differences in the skull size of the participants. The MCA sends blood to the anterior, middle, and posterior temporal arteries, the anterior and posterior parietal arteries, and the lateral orbitofrontal and ascending frontal arteries (Netter, 1989), and thus profuses approximately $80 \%$ of the brain (Stroobant \& Vingerhoets, 2000). Hemovelocity is measured in centimeters/second and is usually time-averaged across a specified time interval. Fast Fourier transformation analysis produces a visual display that presents the three aspects of the data: Velocity is on the vertical axis, time is on the horizontal axis, and the signal intensity is displayed in the multiple colors. The line of the display follows the cardiac cycle and corresponds to the maximum velocity in each cardiac cycle. Experimenters used this visual display to verify the signal.

After securing the signal, the participant stared at the blank computer screen for $2 \mathrm{~min}$, during which BFV was recorded. The 2 min of recorded hemovelocity was the baseline of BFV for the participant. The participants were instructed to relax comfortably with no talking during the baseline recording.

Calibration. Facing the wall, participants were asked to calibrate a laser-modified handgun by shooting nine red dots that appeared around the projection on the wall. The projection provided a visual field of approximately $45^{\circ}$ ( $2.3 \mathrm{~m}$ diagonal). Each dot had to be hit with a laser shot from the laser-modified handgun in any order. This also served to familiarize the participant with the firearm and to provide marksmanship practice. If necessary, the calibration sequence was repeated; however, most participants needed this simple practice only once to be competent.

Peacekeeper task. The Peacekeeper task required an S/DS decision to discriminate between targets and nontargets. Participants monitored a scenic display that simulated a sentry-duty vigilance task. The experiment was presented on Dell computers using software written specifically for the experiment. The display that participants monitored was of the same visual field and size as the calibration sequence (approximately $45^{\circ} ; 2.3 \mathrm{~m}$ diagonal) and was a picture of an Iraqi street scene. The task required searching for threat stimuli that appeared in, on, or around the pictured buildings (see Figure 1B). Threat and nonthreat stimuli were multicolored bull's-eyes, where the center color determined the threat status: Threat stimuli contained black centers, whereas nontargets had blue or red centers. To respond to stimuli that were projected on a wall, targets were shot with a lasermodified handgun. LaserShot (www.lasershot.com) hardware and software automatically recorded the output of a laser-modified handgun, recording both the time and the location of each response.

Each experiment contained 400 trials during the course of the vigilance task, and the task lasted approximately $37 \mathrm{~min}$. Targets (black-centered bull's-eyes) were presented randomly within a sequence of distractor or nonthreat stimuli, which were three times more likely to appear. Each stimulus presentation time varied from
A

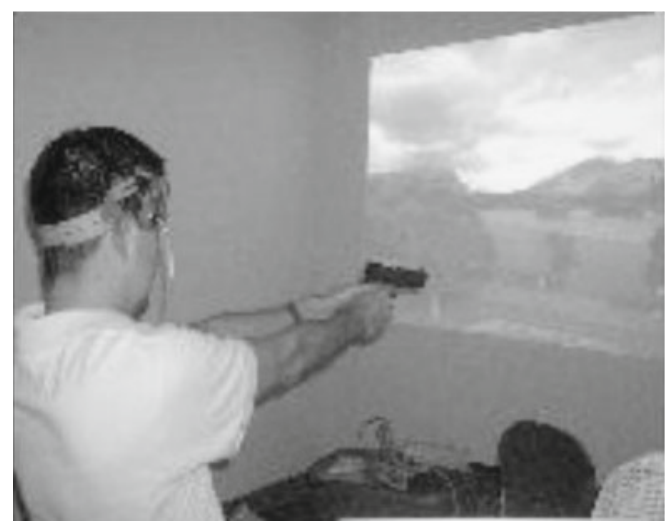

B

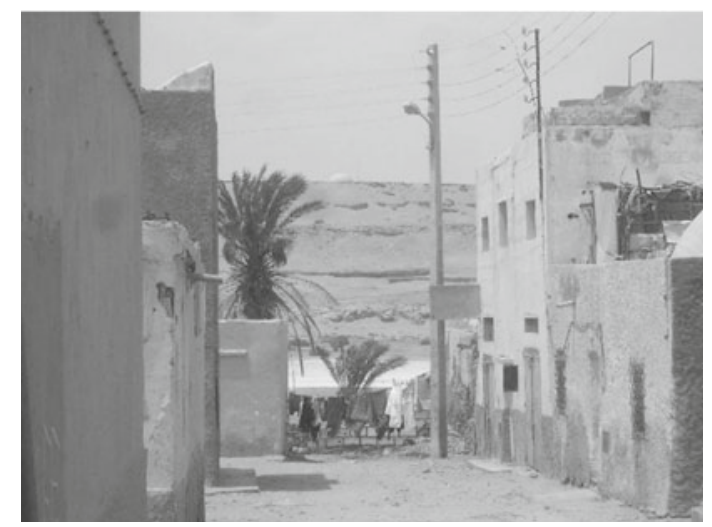

Figure 1. (A) A participant wearing the transcranial Doppler apparatus makes a shoot/don't-shoot decision. (B) The background image used in the present study. Bull's-eye threat and nonthreat images were superimposed on the background (e.g., in the windows, doorways, or corners). 
1,000 to $1,500 \mathrm{msec}$. The amount of time between stimulus presentations also randomly varied from 1 to $5 \mathrm{sec}$.

\section{RESULTS}

\section{Peacekeeper Performance Data}

Performance data were calculated by dividing the task into four successive blocks, each containing 100 trials, of which approximately 33 were target-present and lasted just over 9 min. A repeated measures one-way ANOVA with task period as a within-subjects factor (4 blocks) was used to analyze hit rate, reaction time, and marksmanship separately. The main effect of task period was significant for hit rate $[F(3,420)=9.09, p<.01]$. Post hoc analysis revealed that hit rate was significantly higher in Block $1(M=0.623$, $S D=0.164)$ than in Block $3(M=0.589, S D=0.196)$ or Block 4 $(M=0.569, S D=0.210 ; p=.055)$. Block $2(M=$ $0.621, S D=0.190)$ had a significantly higher hit rate than Block 3 or Block 4. No significant difference was found between the hit rates of Blocks 3 and 4. The main effect of task period was significant for reaction time $[F(3,420)=$ $7.37, p<.01]$. Post hoc analysis revealed that Block 1 had the fastest reaction time and was significantly faster than Block 2, 3, or 4. Block 2 approached significance faster than Block $3(p=.064)$, and Block 2 was significantly faster than Block 4. The main effect of task period was significant for marksmanship $[F(3,420)=7.83, p<.01]$. In the post hoc analysis, marksmanship accuracy was significantly better in Block 1 than in Blocks 3 and 4, with a similar trend for Block $2(p<.07)$. Block 2 had better marksmanship than Blocks 3 and 4 .

\section{TCD Data}

Brain activation was measured through mean cerebral BFV through the MCA. The baseline average was calculated by averaging the BFV from 2 min of recording. Task BFV was calculated by subtracting the baseline average $\mathrm{BFV}$ from the BFV found during the task. Therefore, in the following analyses, all BFV reported was the change in BFV from baseline.

A $4 \times 2$ (block $\times$ hemisphere) repeated measures ANOVA showed a main effect of block $[F(3,147)=50.90$, $p<.01]$ and a main effect of hemisphere $[F(1,49)=4.43$, $p<.05]$. The left hemisphere had significantly faster BFV $(M=0.67, S D=5.87)$ than the right hemisphere $(M=$ $-1.09, S D=6.07)$. A post hoc analysis showed that BFV decreased steadily across the task, such that it was significantly fastest in Block $1(M=2.13, S D=4.99)$, slowing in Block $2(M=-0.18, S D=5.10)$, further slowing in Block $3(M=-0.94, S D=5.49)$, and slowest in the final block, Block $4(M=-1.86, S D=5.94)$. All blocks were significantly different from each other at the $p<.01$ level.

A $2 \times 2$ (hemisphere $\times$ hit $/$ miss) repeated measures ANOVA showed a main effect of hit $/$ miss $[F(1,49)=$ $4.80, p<.05]$, which also interacted significantly with hemisphere $[F(1,49)=11.88, p<.01]$. Post hoc analyses revealed a significant difference between BFV in the left versus right hemisphere at the moment a target was being hit, but not when it was being missed.
A hierarchical regression was run to determine the measures that predicted performance. Each step included predicted a significant amount of the variance in reaction time. Rather than conducting a separate analysis for each individual participant, all the trials were analyzed, and the first predictor included was subject number, to account for individual differences. For reaction time, approximately $13 \%$ of the variance was explained by a model that added the latency of the previous response, vigilance period, and left-hemisphere mean BFV as early as $18 \mathrm{sec}$ before the target presentation. Previous trial reaction time predicted reaction time above and beyond individual differences $\left(\Delta R^{2}=.098, p<.01\right)$. Vigilance period predicted reaction time above and beyond individual differences and previous reaction time $\left(\Delta R^{2}=.024, p<.01\right)$. BFV $18 \mathrm{sec}$ before the target presentation predicted reaction time above and beyond individual differences, previous reaction time, and vigilance period $\left(\Delta R^{2}=.015, p<.01\right)$.

\section{CONCLUSIONS}

This study was designed to test whether TCD could predict variations in S/DS performance. Replicating and extending previous research, a vigilance decrement was found in the Peacekeeper task. The findings display a significant decrease in task performance across the vigilance task for all behavioral measures. Hit rate decreased across the task, as did accuracy as measured by marksmanship. Reaction time increased across the task (i.e., participants took longer to hit the targets). Although the Peacekeeper task was more interactive, focusing more on decision making in an S/DS scenario, the behavior data were expected to decrease as they did, and all the findings were in line with previous vigilance research.

The addition of the physiological measure of cerebral BFV added to an alternate form of measuring the level of attention during the task. BFV decreased across the task, also showing the vigilance decrement effects, such that BFV in each successive block was significantly lower than in the previous block. These changes in cerebral BFV added significantly (albeit not greatly) to the prediction of changes in vigilance. Additionally, BFV differed according to whether the target was hit or missed. For target hits, BFV was significantly higher in the left hemisphere than the right, whereas for missed targets no significant difference was found between hemispheres.

In the Peacekeeper task, the left hemisphere was found to be dominant, which was contradictory to the expectations and previous research. Previous vigilance studies have had findings of right-hemisphere dominance (Helton et al., 2007; Hitchcock et al., 2003; Warm \& Parasuraman, 2007). Although the Peacekeeper task is a vigilance task, other aspects could be causing the lefthemisphere lateralization. Performance was focused also on the S/DS decision making and marksmanship with the laser-modified handgun. Additionally, display times and intertrial intervals were randomized. Any of these elements could be the cause of the switch in lateralization. This task is not a traditional vigilance task; thus, future 
research will have to determine which characteristic of the task is engaging the left hemisphere over the right hemisphere. However, our findings do indicate that righthemisphere lateralization found previously in vigilance research may be a function of the characteristics of the tasks instead of a signature of the brain activation during a period of vigilance.

\section{AUTHOR NOTE}

This research was supported by a grant from the United States Army Medical Corp and Materiel Command. Additional support was provided by the National Institute of Child Health and Human Development (HD 38051). The authors thank Jared Taglialatela, who wrote the software for this task, and the research assistants in the Individual Differences in Executive Attention (IDEA) laboratory at Georgia State University, who provided critical assistance in data collection. Correspondence concerning this article should be addressed to D. A. Washburn, Department of Psychology, Georgia State University, Box 5010, Atlanta, GA 303025010 (e-mail: dwashburn@gsu.edu).

\section{REFERENCES}

Aaslid, R., Markwalder, T. M., \& Nornes, H. (1982). Noninvasive transcranial Doppler ultrasound recording of flow velocity in basal cerebral arteries. Journal of Neurosurgery, 57, 769-774.

Angevine, J. B., \& Cotman, C. W. (1981). Principles of neuroanatomy. New York: New York University Press.

Bulla-Hellwig, M., Vollmer, J., Götzen, A., Skreczek, W., \& HartJe, W. (1996). Hemispheric asymmetry of arterial blood flow velocity changes during verbal and visuospatial tasks. Neuropsychologia, 34, 987-991.

CARPENTER, M. B. (1976). Human neuroanatomy (7th ed.). Baltimore: Williams \& Wilkins.

Cupini, L. M., Matteis, M., Troisi, E., Sabbadini, M., Bernardi, G., Caltagirone, C., \& Silvestrini, M. (1996). Bilateral simultaneous transcranial Doppler monitoring of flow velocity changes during visuospatial and verbal working memory tasks. Brain, 119, 1249-1253.

DuscheK, S., \& SchANDRY, R. (2003). Functional transcranial Doppler sonography as a tool in psychophysiological research. Psychophysiology, 40, 436-454.

Hanaway, J., Woolsey, T. A., Gado, M. H., \& Roberts, M. P., JR. (1998). The brain atlas: A visual guide to the human central nervous system. Bethesda, MD: Fitzgerald Science Press.

Helton, W. S., Dember, W. N., Warm, J. S., \& Matthews, G. (1999).
Optimism, pessimism, and false failure feedback: Effects on vigilance performance. Current Psychology, 18, 311-325.

Helton, W. S., Hollander, T. D., Warm, J. S., Tripp, L. D., Parsons, K., Matthews, G., ET AL. (2007). The abbreviated vigilance task and cerebral hemodynamics. Journal of Clinical \& Experimental Neuropsychology, 29, 545-552.

Hitchсоск, E. M., Warm, J. S., Matthews, G., Dember, W. N., Shear, P. K., TRIPP, L. D., ET AL. (2003). Automation cueing modulates cerebral blood flow and vigilance in a simulated air traffic control task. Theoretical Issues in Ergonomics Science, 4, 89-112.

MackWORTH, N. H. (1961). Researches on the measurement of human performance. In H. W. Sinaiko (Ed.), Selected papers in the design and use of control systems (pp. 174-331). New York: Dover. (Reprinted from Medical Research Council Special Report Series 268, 1950, London: HM Stationary Office).

NetTer, F. H. (1989). Atlas of human anatomy. Summit, NJ: CibaGeigy Corp.

OldFIELD, R. C. (1971). The assessment and analysis of handedness: The Edinburgh inventory. Neuropsychologia, 9, 97-113.

Parasuraman, R. (Ed.) (2000). The attentive brain. Cambridge, MA: MIT Press.

Parasuraman, R., \& Davies, D. R. (Eds.) (1984). Varieties of attention. Orlando, FL: Academic Press.

Schnittger, C., Johannes, S., Arnavaz, A., \& Munte, T. F. (1997). Relation of cerebral blood flow velocity and level of vigilance in humans. NeuroReport, 8, 1637-1639.

See, J. E., Howe, S. R., Warm, J. S., \& Dember, W. N. (1995). Metaanalysis of the sensitivity decrement in vigilance. Psychological Bulletin, 117, 230-249.

Silvestrini, M., Cupini, L. M., Matteis, M., Troisi, E., \& CaltagiRONE, C. (1994). Bilateral simultaneous assessment of cerebral flow velocity during mental activity. Journal of Cerebral Blood Flow \& Metabolism, 14, 643-648.

Stroobant, N., \& Vingerhoets, G. (2000). Transcranial Doppler ultrasonography monitoring of cerebral hemodynamics during performance of cognitive tasks: A review. Neuropsychology Review, 10, 213-231.

Teichner, W. H. (1974). The detection of a simple visual signal as a function of time on watch. Human Factors, 16, 339-353.

Warm, J. S., \& Parasuraman, R. (2007). Cerebral hemodynamics and vigilance. In R. Parasuraman \& M. Rizzo (Eds.), Neuroergonomics: The brain at work (pp. 146-158). New York: Oxford University Press.

(Manuscript received December 6, 2007; revision accepted for publication May 8, 2008.) 